Article

\title{
Integral Inequalities of Hermite-Hadamard Type for Extended s-Convex Functions and Applications
}

\author{
Ye Shuang ${ }^{1}$ and Feng $\mathrm{Qi}^{2,3, *}$ (i) \\ 1 College of Mathematics, Inner Mongolia University for Nationalities, Tongliao, Inner Mongolia 028043, \\ China; shuangye152300@sina.com \\ 2 Institute of Mathematics, Henan Polytechnic University, Jiaozuo 454010, China \\ 3 Department of Mathematics, College of Science, Tianjin Polytechnic University, Tianjin 300387, China \\ * Correspondence: qifeng618@gmail.com
}

Received: 6 September 2018; Accepted: 24 October 2018; Published: 28 October 2018

Abstract: In the paper, the authors set up an identity for a function whose third derivative is integrable, establish by the Hölder inequality some new integral inequalities of the Hermite-Hadamard type for extended s-convex functions in the second sense, and apply these integral inequalities to construct inequalities for several special means.

Keywords: extended s-convex function in the second sense; Hermite-Hadamard type inequality; Hölder inequality; mean

MSC: 26A51; 26D15; 26D20; 26E60; 41A55

\section{Introduction}

A function $h: I \subseteq \mathbb{R} \rightarrow \mathbb{R}$ is said to be convex if

$$
h(\mu u+(1-\mu) v) \leq \mu h(u)+(1-\mu) h(v)
$$

holds for all $u, v \in I$ and $\mu \in[0,1]$. For any convex function $h(t)$ on a closed interval $[a, b]$, the double inequality

$$
h\left(\frac{a+b}{2}\right) \leq \int_{a}^{b} h(t) \mathrm{d} t \leq \frac{h(a)+h(b)}{2}
$$

is classical and called as the Hermite-Hadamard integral inequality.

It is well known that the convex function is a basic concept in mathematics and mathematical sciences and there exists a theory on convex functions. For the theory of convex functions, please refer to two monographs [1,2], for example. Among the theory of convex functions, the Hermite-Hadamard type inequalities play important roles. For new development of the Hermite-Hadamard type inequalities, please refer to the monograph [3], for example.

We now introduce the s-convex function in the second sense and the extended s-convex function in the second sense.

Definition $1([4])$. Let $s \in(0,1]$. A function $h: I \subseteq[0, \infty) \rightarrow \mathbb{R}$ is said to be s-convex in the second sense if

$$
h(\mu u+(1-\mu) v) \leq \mu^{s} h(u)+(1-\mu)^{s} h(v)
$$

holds for all $u, v \in I$ and $\mu \in[0,1]$. 
Definition 2 ([5]). For some $s \in[-1,1]$, a function $h: I \subseteq[0, \infty) \rightarrow \mathbb{R}$ is said to be extended s-convex in the second sense if the inequality (2) holds for all $u, v \in I$ and $\mu \in[0,1]$.

Remark 1. We note that, the following five cases, and only the following five cases, are possible:

1. when $s \in(0,1]$, any s-convex function in the second sense is equivalent to an extended s-convex function in the second sense for the same $s \in(0,1]$;

2. when $s \in(0,1]$ and $h(t)$ is an s-convex function in the second sense, there exists a number $s_{0} \in[-1,1] \backslash\{s\}$ such that $h(t)$ is an extended $s_{0}$-convex function in the second sense;

3. when $s \in(0,1]$ and $h(t)$ is an s-convex function in the second sense, there does not exist a number $s_{0} \in[-1,1] \backslash\{s\}$ such that $h(t)$ is an extended $s_{0}$-convex function in the second sense; equivalently speaking, for any $s \in(0,1]$, there exists an s-convex function $h(t)$ in the second sense, but $h(x)$ is not an $s_{0}$-convex function in the second sense for all $s_{0} \in[-1,1] \backslash\{s\}$;

4. when $s \in(0,1]$ and $h(t)$ is not an s-convex function in the second sense, there exists a number $s_{0} \in[-1,1] \backslash\{s\}$ such that $h(t)$ is an extended $s_{0}$-convex function in the second sense;

5. when $s \in(0,1]$ and $h(t)$ is not an s-convex function in the second sense, there does not exist a number $s_{0} \in[-1,1] \backslash\{s\}$ such that $h(t)$ is an extended $s_{0}$-convex function in the second sense; equivalently speaking, for any $s \in(0,1]$, there exists a function $h(t)$ which is neither an s-convex function in the second sense nor an extended $s_{0}$-convex function in the second sense for all $s_{0} \in[-1,1] \backslash\{s\}$.

We now construct an example as follows. Let $h(t)=1-t^{2}$ for $t \in[0,1]$. Taking $\mu=0.1, u=0.8$, $v=0.4$, and $s \in(0,1]$ in Definition 2 yields

$$
h(\mu u+(1-\mu) v)-\left[\mu^{s} h(u)+(1-\mu)^{s} h(v)\right]=0.8064-\left[0.36 \times 0.1^{s}+0.84 \times 0.9^{s}\right]>0 .
$$

This means that $h(t)$ is not an s-convex function in the second sense on $[0,1]$ for $s \in(0,1]$. On the other hand, it is easy to see that

$$
(1-\mu)\left(1-u^{2}\right)+\mu\left(1-v^{2}\right)-\mu(1-\mu)\left\{1-[\mu u+(1-\mu) v]^{2}\right\} \geq 0
$$

for every $u, v \in[0,1]$ and $\mu \in(0,1)$. This means that $h(t)$ is an extended-1-convex function in the second sense on $[0,1]$.

There have been some studies dedicated to generalizing the s-convex function in the second sense and the extended s-convex function in the second sense and to establishing their Hermite-Hadamard type inequalities. For more details, please refer to the papers [6-15] and closely related references therein.

The following are some of the Hermite-Hadamard type inequalities for $s$-convex functions in the second sense.

Theorem 1 ([16]). Let $h: I \subseteq[0, \infty) \rightarrow \mathbb{R}$ be differentiable on $I^{\circ}$ and $a, b \in I$ with $a<b$ and $h^{\prime} \in L_{1}([a, b])$. If $\left|h^{\prime}(t)\right|^{q}$ is s-convex in the second sense on $[a, b]$ for some fixed $s \in(0,1]$ and $q \geq 1$, then

$$
\left|h\left(\frac{a+b}{2}\right)-\frac{1}{b-a} \int_{a}^{b} h(t) \mathrm{d} t\right| \leq \frac{b-a}{2}\left(\frac{1}{2}\right)^{1-1 / q}\left[\frac{2+1 / 2^{s}}{(s+1)(s+2)}\right]^{1 / q}\left[\left|h^{\prime}(a)\right|^{q}+\left|h^{\prime}(b)\right|^{q}\right]^{1 / q} .
$$

Theorem 2 ([17]). Let $h: I \subseteq[0, \infty) \rightarrow \mathbb{R}$ be differentiable on $I^{\circ}, a, b \in I$ with $a<b$, and $h^{\prime} \in L_{1}([a, b])$. If $\left|h^{\prime}(t)\right|^{q}$ is s-convex in the second sense on $[a, b]$ for some fixed $s \in(0,1]$, and $q>1$, then

$$
\begin{aligned}
\mid h\left(\frac{a+b}{2}\right) & -\frac{1}{b-a} \int_{a}^{b} h(t) \mathrm{d} t \mid \leq \frac{b-a}{4}\left[\frac{1}{(s+1)(s+2)}\right]^{1 / q}\left(\frac{1}{2}\right)^{1 / p} \\
& \times\left\{\left[\left|h^{\prime}(a)\right|^{q}+(s+1)\left|h^{\prime}\left(\frac{a+b}{2}\right)\right|^{q}\right]^{1 / q}+\left[\left|h^{\prime}(b)\right|^{q}+(s+1)\left|h^{\prime}\left(\frac{a+b}{2}\right)\right|^{q}\right]^{1 / q}\right\},
\end{aligned}
$$


where $\frac{1}{p}+\frac{1}{q}=1$.

Theorem 3 ([18]). Let $h: I \subseteq[0, \infty) \rightarrow \mathbb{R}$ be differentiable on $I^{\circ}, a, b \in I$ with $a<b$, and $h^{\prime} \in L_{1}([a, b])$. If $\left|h^{\prime}(t)\right|$ is s-convex in the second sense on $[a, b]$ for some fixed $s \in(0,1]$, then

$$
\begin{aligned}
\mid \frac{1}{6}\left[h(a)+h(b)+4 h\left(\frac{a+b}{2}\right)\right] & -\frac{1}{b-a} \int_{a}^{b} h(t) \mathrm{d} t \mid \\
& \leq \frac{(s-4) 6^{s+1}+2 \times 5^{s+2}-2 \times 3^{s+2}+2}{6^{s+2}(s+1)(s+2)}(b-a)\left[\left|h^{\prime}(a)\right|+\left|h^{\prime}(b)\right|\right] .
\end{aligned}
$$

During the past two decades, starting from s-convex functions to generalizing different concepts of convex functions, there has been a continuous interest in the development of integral inequalities of the Hermite-Hadamard type. It seems that it is a rich machinery for obtaining results. For example, in the paper [19] and closely related references therein, a strong extension and refinement of the Hadamard inequality in the right hand side of (1) was generalized to nonlinear integrals. For more results on integral inequalities of the Hermite-Hadamard type for diverse convex functions, please refer to [20-27] and closely related references therein.

In [6], some integral inequalities of the Simpson type were established for a function $h(t)$ whose third derivative $h^{\prime \prime \prime}(t)$ belongs to $L_{1}([a, b])$ and $\left|h^{\prime \prime \prime}(t)\right|^{q}$ is an extended $s$-convex in the second sense for $q \geq 1$. One of the key steps is to set up an identity

$$
\begin{aligned}
\frac{1}{6}\left[h(a)+4 h\left(\frac{a+b}{2}\right)+h(b)\right]-\frac{1}{b-a} \int_{a}^{b} h(t) \mathrm{d} t \\
\quad=\frac{(b-a)^{3}}{96} \int_{0}^{1} t(1-t)^{2}\left[h^{\prime \prime \prime}\left(t b+(1-t) \frac{a+b}{2}\right)-h^{\prime \prime \prime}\left(t a+(1-t) \frac{a+b}{2}\right)\right] \mathrm{d} t
\end{aligned}
$$

for $h: I \subseteq \mathbb{R} \rightarrow \mathbb{R}, a, b \in I$ with $a<b$, and $h^{\prime \prime \prime} \in L_{1}([a, b])$.

In this paper, we will set up an identity different from (3), establish some integral inequalities for a function $h(t)$ whose third derivative $h^{\prime \prime \prime}(t)$ belongs to $L_{1}([a, b])$ and $\left|h^{\prime \prime \prime}(t)\right|^{q}$ is an extended $s$-convex in the second sense for $q \geq 1$, and apply these integral inequalities to construct inequalities for several special means.

\section{A Lemma}

For attaining our main aim, we need a lemma below.

Lemma 1. Let $h: I \subseteq \mathbb{R} \rightarrow \mathbb{R}$ be a three times differentiable function on $I^{\circ}$ and $a, b \in I$ with $a<b$. If $h^{\prime \prime \prime} \in L_{1}([a, b])$ and $\lambda \in[0,1]$, then

$$
\begin{array}{r}
\frac{1}{b-a} \int_{a}^{b} h(t) \mathrm{d} t-\frac{3 \lambda^{2}-3 \lambda+1}{6}(b-a)^{2} h^{\prime \prime}(\lambda a+(1-\lambda) b) \\
-\frac{2 \lambda-1}{2}(b-a) h^{\prime}(\lambda a+(1-\lambda) b)-h(\lambda a+(1-\lambda) b) \\
=\frac{(b-a)^{3}}{6}\left[\lambda^{4} \int_{0}^{1} t^{3} h^{\prime \prime \prime}(t(\lambda a+(1-\lambda) b)+(1-t) b) \mathrm{d} t\right. \\
\left.\quad-(1-\lambda)^{4} \int_{0}^{1} t^{3} h^{\prime \prime \prime}(t(\lambda a+(1-\lambda) b)+(1-t) a) \mathrm{d} t\right] .
\end{array}
$$

Proof. When $\lambda \in(0,1)$, by integrating by parts, we have

$$
\int_{0}^{1} t^{3} h^{\prime \prime \prime}(t(\lambda a+(1-\lambda) b)+(1-t) b) \mathrm{d} t
$$




$$
\begin{aligned}
= & -\frac{1}{\lambda(b-a)} \int_{0}^{1} t^{3} \mathrm{~d} h^{\prime \prime}(t(\lambda a+(1-\lambda) b)+(1-t) b) \\
= & -\frac{1}{\lambda(b-a)} h^{\prime \prime}(\lambda a+(1-\lambda) b)-\frac{3}{\lambda^{2}(b-a)^{2}} \int_{0}^{1} t^{2} \mathrm{~d} h^{\prime}(t(\lambda a+(1-\lambda) b)+(1-t) b) \\
= & -\frac{1}{\lambda(b-a)} h^{\prime \prime}(\lambda a+(1-\lambda) b)-\frac{3}{\lambda^{2}(b-a)^{2}} h^{\prime}(\lambda a+(1-\lambda) b) \\
& -\frac{6}{\lambda^{3}(b-a)^{3}} \int_{0}^{1} t \mathrm{~d} h(t(\lambda a+(1-\lambda) b)+(1-t) b) \\
= & -\frac{1}{\lambda(b-a)} h^{\prime \prime}(\lambda a+(1-\lambda) b)-\frac{3}{\lambda^{2}(b-a)^{2}} h^{\prime}(\lambda a+(1-\lambda) b)-\frac{6}{\lambda^{3}(b-a)^{3}} h(\lambda a+(1-\lambda) b) \\
& +\frac{6}{\lambda^{3}(b-a)^{3}} \int_{0}^{1} h(t(\lambda a+(1-\lambda) b)+(1-t) b) \mathrm{d} t
\end{aligned}
$$

and

$$
\begin{aligned}
& \int_{0}^{1} t^{3} h^{\prime \prime \prime}(t(\lambda a+(1-\lambda) b)+(1-t) a) \mathrm{d} t \\
= & \frac{1}{(1-\lambda)(b-a)} \int_{0}^{1} t^{3} \mathrm{~d} h^{\prime \prime}(t(\lambda a+(1-\lambda) b)+(1-t) a) \\
= & \frac{1}{(1-\lambda)(b-a)} h^{\prime \prime}(\lambda a+(1-\lambda) b)-\frac{3}{(1-\lambda)^{2}(b-a)^{2}} \int_{0}^{1} t^{2} \mathrm{~d} h^{\prime}(t(\lambda a+(1-\lambda) b)+(1-t) a) \\
= & \frac{1}{(1-\lambda)(b-a)} h^{\prime \prime}(\lambda a+(1-\lambda) b)-\frac{3}{(1-\lambda)^{2}(b-a)^{2}} h^{\prime}(\lambda a+(1-\lambda) b) \\
& +\frac{6}{(1-\lambda)^{3}(b-a)^{3}} \int_{0}^{1} t \mathrm{~d} h(t(\lambda a+(1-\lambda) b)+(1-t) a) \\
= & \frac{1}{(1-\lambda)(b-a)} h^{\prime \prime}(\lambda a+(1-\lambda) b)-\frac{3}{(1-\lambda)^{2}(b-a)^{2}} h^{\prime}(\lambda a+(1-\lambda) b) \\
& +\frac{6}{(1-\lambda)^{3}(b-a)^{3}} h(\lambda a+(1-\lambda) b)-\frac{6}{(1-\lambda)^{3}(b-a)^{3}} \int_{0}^{1} h(t(\lambda a+(1-\lambda) b)+(1-t) a) \mathrm{d} t .
\end{aligned}
$$

Substituting these two equalities into the right side of (4) and changing variables result in the required conclusion. The proof is complete.

Remark 2. When taking $\lambda=0,1$ in (4) respectively, we derive

$$
\frac{1}{b-a} \int_{a}^{b} h(t) \mathrm{d} t-\frac{1}{6}(b-a)^{2} h^{\prime \prime}(b)+\frac{1}{2}(b-a) h^{\prime}(b)-h(b)=-\frac{(b-a)^{3}}{6} \int_{0}^{1} t^{3} h^{\prime \prime \prime}(t b+(1-t) a) \mathrm{d} t
$$

and

$$
\frac{1}{b-a} \int_{a}^{b} h(t) \mathrm{d} t-\frac{1}{6}(b-a)^{2} h^{\prime \prime}(a)-\frac{1}{2}(b-a) h^{\prime}(a)-h(a)=\frac{(b-a)^{3}}{6} \int_{0}^{1} t^{3} h^{\prime \prime \prime}(t a+(1-t) b) \mathrm{d} t
$$

respectively.

The identity (3) mentioned above and the newly-established identity (4) can not be derived from each other.

\section{Inequalities for Extended $s$-Convex Functions in the Second Sense}

Now, we are in a position to establish some new integral inequalities of the Hermite-Hadmard type for extended s-convex functions in the second sense.

Theorem 4. Let $h: I \subseteq[0, \infty) \rightarrow \mathbb{R}$ be a three times differentiable function on $I, a, b \in I$ with $a<b$, $h^{\prime \prime \prime} \in L_{1}([a, b])$, and $\lambda \in[0,1]$. If $\left|h^{\prime \prime \prime}(t)\right|^{q}$ is an extended s-convex function in the second sense on $[a, b]$, $s \in[-1,1]$, and $q \geq 1$, then

1. when $\lambda \in[0,1]$ and $-1<s \leq 1$, we have 


$$
\begin{aligned}
\mid \frac{1}{b-a} \int_{a}^{b} h(t) \mathrm{d} t-\frac{3 \lambda^{2}-3 \lambda+1}{6}(b-a)^{2} h^{\prime \prime}(\lambda a+(1-\lambda) b) & -\frac{2 \lambda-1}{2}(b-a) h^{\prime}(\lambda a+(1-\lambda) b)-h(\lambda a+(1-\lambda) b) \mid \leq \frac{(b-a)^{3}}{24} \\
\times & {\left[\frac{4}{(s+1)(s+2)(s+3)(s+4)}\right]^{1 / q}\left\{( 1 - \lambda ) ^ { 4 } \left[(s+1)(s+2)(s+3)\left|h^{\prime \prime \prime}(\lambda a+(1-\lambda) b)\right|^{q}\right.\right.} \\
+ & \left.\left.6\left|h^{\prime \prime \prime}(a)\right|^{q}\right]^{1 / q}+\lambda^{4}\left[(s+1)(s+2)(s+3)\left|h^{\prime \prime \prime}(\lambda a+(1-\lambda) b)\right|^{q}+6\left|h^{\prime \prime \prime}(b)\right|^{q}\right]^{1 / q}\right\}
\end{aligned}
$$

2. when $\lambda \in(0,1)$ and $s=-1$, we have

$$
\begin{aligned}
& \mid \frac{1}{b-a} \int_{a}^{b} h(t) \mathrm{d} t-\frac{3 \lambda^{2}-3 \lambda+1}{6}(b-a)^{2} h^{\prime \prime}(\lambda a+(1-\lambda) b) \\
&-\frac{2 \lambda-1}{2}(b-a) h^{\prime}(\lambda a+(1-\lambda) b)-h(\lambda a+(1-\lambda) b) \mid \leq \frac{(b-a)^{3}}{24}\left[\frac{2}{3 \lambda^{4}(1-\lambda)^{4}}\right]^{1 / q} \\
& \times\left\{(1-\lambda)^{4}\left[2(1-\lambda)^{3} \lambda^{4}\left|h^{\prime \prime \prime}(b)\right|^{q}-\lambda^{4}\left(2(1-\lambda)^{3}+3(1-\lambda)^{2}+6(1-\lambda)+6 \ln \lambda\right)\left|h^{\prime \prime \prime}(a)\right|^{q}\right]^{1 / q}\right. \\
&\left.\quad+\lambda^{4}\left[2(1-\lambda)^{4} \lambda^{3}\left|h^{\prime \prime \prime}(a)\right|^{q}-(1-\lambda)^{4}\left(2 \lambda^{3}+3 \lambda^{2}+6 \lambda+6 \ln (1-\lambda)\right)\left|h^{\prime \prime \prime}(b)\right|^{q}\right]^{1 / q}\right\} .
\end{aligned}
$$

Proof. When $\lambda \in[0,1]$ and $-1<s \leq 1$, since $\left|h^{\prime \prime \prime}(t)\right|^{q}$ is an extended $s$-convex function in the second sense on $[a, b]$, by Lemma 1 and the Hölder inequality, we have

$$
\begin{aligned}
\mid & \frac{1}{b-a} \int_{a}^{b} h(t) \mathrm{d} t-\frac{3 \lambda^{2}-3 \lambda+1}{6}(b-a)^{2} h^{\prime \prime}(\lambda a+(1-\lambda) b) \\
& -\frac{2 \lambda-1}{2}(b-a) h^{\prime}(\lambda a+(1-\lambda) b)-h(\lambda a+(1-\lambda) b) \mid \\
\leq & \frac{(b-a)^{3}}{6}\left[\lambda^{4} \int_{0}^{1}\left|t^{3} h^{\prime \prime \prime}(t(\lambda a+(1-\lambda) b)+(1-t) b)\right| \mathrm{d} t\right. \\
& \left.+(1-\lambda)^{4} \int_{0}^{1}\left|t^{3} h^{\prime \prime \prime}(t(\lambda a+(1-\lambda) b)+(1-t) a)\right| \mathrm{d} t\right] \\
\leq & \frac{(b-a)^{3}}{6}\left\{\lambda^{4}\left(\int_{0}^{1} t^{3} \mathrm{~d} t\right)^{1-1 / q}\left[\int_{0}^{1} t^{3}\left|h^{\prime \prime \prime}(t(\lambda a+(1-\lambda) b)+(1-t) b)\right|^{q} \mathrm{~d} t\right]^{1 / q}\right. \\
& \left.+(1-\lambda)^{4}\left(\int_{0}^{1} t^{3} \mathrm{~d} t\right)^{1-1 / q}\left[\int_{0}^{1} t^{3}\left|h^{\prime \prime \prime}(t(\lambda a+(1-\lambda) b)+(1-t) a)\right|^{q} \mathrm{~d} t\right]^{1 / q}\right\} \\
\leq & \frac{(b-a)^{3}}{6}\left\{\lambda^{4}\left(\int_{0}^{1} t^{3} \mathrm{~d} t\right)^{1-1 / q}\left[\int_{0}^{1} t^{3}\left(t^{s}\left|h^{\prime \prime \prime}(\lambda a+(1-\lambda) b)\right|^{q}+(1-t)^{s}\left|h^{\prime \prime \prime}(b)\right|^{q}\right) \mathrm{d} t\right]^{1 / q}\right. \\
& \left.+(1-\lambda)^{4}\left(\int_{0}^{1} t^{3} \mathrm{~d} t\right)^{1-1 / q}\left[\int_{0}^{1} t^{3}\left(t^{s}\left|h^{\prime \prime \prime}(\lambda a+(1-\lambda) b)\right|^{q}+(1-t)^{s}\left|h^{\prime \prime \prime}(a)\right|^{q}\right) \mathrm{d} t\right]^{1 / q}\right\} \\
= & \frac{(b-a)^{3}}{6}\left\{\lambda^{4}\left(\frac{1}{4}\right)^{1-1 / q}\left[\frac{\left|h^{\prime \prime \prime}(\lambda a+(1-\lambda) b)\right|^{q}}{s+4}+\frac{6\left|h^{\prime \prime \prime}(b)\right|^{q}}{(s+1)(s+2)(s+3)(s+4)}\right]^{1 / q}\right. \\
& \left.+(1-\lambda)^{4}\left(\frac{1}{4}\right)^{1-1 / q}\left[\frac{\left|h^{\prime \prime \prime}(\lambda a+(1-\lambda) b)\right|^{q}}{s+4}+\frac{6\left|h^{\prime \prime \prime}(a)\right|^{q}}{(s+1)(s+2)(s+3)(s+4)}\right]^{1 / q}\right\} \\
= & \frac{(b-a)^{3}}{24}\left[\frac{4}{(s+1)(s+2)(s+3)(s+4)}\right]^{1 / q}\left\{( 1 - \lambda ) ^ { 4 } \left[(s+1)(s+2)(s+3)\left|h^{\prime \prime \prime}(\lambda a+(1-\lambda) b)\right|^{q}\right.\right. \\
& \left.\left.+6\left|h^{\prime \prime \prime}(a)\right|^{q}\right]^{1 / q}+\lambda^{4}\left[(s+1)(s+2)(s+3)\left|h^{\prime \prime \prime}(\lambda a+(1-\lambda) b)\right|^{q}+6\left|h^{\prime \prime \prime}(b)\right|^{q}\right]^{1 / q}\right\} .
\end{aligned}
$$

When $\lambda \in(0,1)$ and $s=-1$, since $\left|h^{\prime \prime \prime}(t)\right|^{q}$ is an extended $s$-convex function in the second sense on $[a, b]$, by Lemma 1 and the Hölder inequality, we have

$$
\begin{aligned}
& \mid \frac{1}{b-a} \int_{a}^{b} h(t) \mathrm{d} t-\frac{3 \lambda^{2}-3 \lambda+1}{6}(b-a)^{2} h^{\prime \prime}(\lambda a+(1-\lambda) b) \\
& -\frac{2 \lambda-1}{2}(b-a) h^{\prime}(\lambda a+(1-\lambda) b)-h(\lambda a+(1-\lambda) b) \mid
\end{aligned}
$$




$$
\begin{aligned}
& \leq \frac{(b-a)^{3}}{6}\left[\lambda^{4} \int_{0}^{1}\left|t^{3} h^{\prime \prime \prime}(t(\lambda a+(1-\lambda) b)+(1-t) b)\right| \mathrm{d} t\right. \\
& \left.+(1-\lambda)^{4} \int_{0}^{1}\left|t^{3} h^{\prime \prime \prime}(t(\lambda a+(1-\lambda) b)+(1-t) a)\right| \mathrm{d} t\right] \\
& \leq \frac{(b-a)^{3}}{6}\left\{\lambda^{4}\left(\int_{0}^{1} t^{3} \mathrm{~d} t\right)^{1-1 / q}\left[\int_{0}^{1} t^{3}\left((t \lambda)^{-1}\left|h^{\prime \prime \prime}(a)\right|^{q}+(1-t \lambda)^{-1}\left|h^{\prime \prime \prime}(b)\right|^{q}\right) \mathrm{d} t\right]^{1 / q}\right. \\
& \left.+(1-\lambda)^{4}\left(\int_{0}^{1} t^{3} \mathrm{~d} t\right)^{1-1 / q}\left[\int_{0}^{1} t^{3}\left((t \lambda+1-t)^{-1}\left|h^{\prime \prime \prime}(a)\right|^{q}+(t-t \lambda)^{-1}\left|h^{\prime \prime \prime}(b)\right|^{q}\right) \mathrm{d} t\right]^{1 / q}\right\} \\
& =\frac{(b-a)^{3}}{6}\left\{\lambda^{4}\left(\frac{1}{4}\right)^{1-1 / q}\left[\frac{1}{3 \lambda}\left|h^{\prime \prime \prime}(a)\right|^{q}-\frac{2 \lambda^{3}+3 \lambda^{2}+6 \lambda+6 \ln (1-\lambda)}{6 \lambda^{4}}\left|h^{\prime \prime \prime}(b)\right|^{q}\right]^{1 / q}\right. \\
& \left.+(1-\lambda)^{4}\left(\frac{1}{4}\right)^{1-1 / q}\left[\frac{\left|h^{\prime \prime \prime}(b)\right|^{q}}{3(1-\lambda)}-\frac{2(1-\lambda)^{3}+3(1-\lambda)^{2}+6(1-\lambda)+6 \ln \lambda}{6(1-\lambda)^{4}}\left|h^{\prime \prime \prime}(a)\right|^{q}\right]^{1 / q}\right\} \\
& =\frac{(b-a)^{3}}{24}\left[\frac{2}{3 \lambda^{4}(1-\lambda)^{4}}\right]^{1 / q}\left\{( 1 - \lambda ) ^ { 4 } \left[2(1-\lambda)^{3} \lambda^{4}\left|h^{\prime \prime \prime}(b)\right|^{q}-\lambda^{4}\left(2(1-\lambda)^{3}+3(1-\lambda)^{2}\right.\right.\right. \\
& \left.+6(1-\lambda)+6 \ln \lambda)\left|h^{\prime \prime \prime}(a)\right|^{q}\right]^{1 / q}+\lambda^{4}\left[2(1-\lambda)^{4} \lambda^{3}\left|h^{\prime \prime \prime}(a)\right|^{q}-(1-\lambda)^{4}\left(2 \lambda^{3}+3 \lambda^{2}\right.\right. \\
& \left.\left.+6 \lambda+6 \ln (1-\lambda))\left|h^{\prime \prime \prime}(b)\right|^{q}\right]^{1 / q}\right\} \text {. }
\end{aligned}
$$

The proof of Theorem 4 is complete.

Corollary 1. If $s=1$ in (5), then

$$
\begin{aligned}
\mid \frac{1}{b-a} \int_{a}^{b} h(t) \mathrm{d} t- & \frac{3 \lambda^{2}-3 \lambda+1}{6}(b-a)^{2} h^{\prime \prime}(\lambda a+(1-\lambda) b) \\
& -\frac{2 \lambda-1}{2}(b-a) h^{\prime}(\lambda a+(1-\lambda) b)-h(\lambda a+(1-\lambda) b) \mid \leq \frac{(b-a)^{3}}{24}\left(\frac{1}{5}\right)^{1 / q} \\
& \times\left\{(1-\lambda)^{4}\left[4\left|h^{\prime \prime \prime}(\lambda a+(1-\lambda) b)\right|^{q}+\left|h^{\prime \prime \prime}(a)\right|^{q}\right]^{1 / q}+\lambda^{4}\left[4\left|h^{\prime \prime \prime}(\lambda a+(1-\lambda) b)\right|^{q}+\left|h^{\prime \prime \prime}(b)\right|^{q}\right]^{1 / q}\right\} .
\end{aligned}
$$

Corollary 2. If $s=q=1$ in (5), then

$$
\begin{aligned}
\mid \frac{1}{b-a} \int_{a}^{b} h(t) \mathrm{d} t- & \frac{3 \lambda^{2}-3 \lambda+1}{6}(b-a)^{2} h^{\prime \prime}(\lambda a+(1-\lambda) b) \\
& -\frac{2 \lambda-1}{2}(b-a) h^{\prime}(\lambda a+(1-\lambda) b)-h(\lambda a+(1-\lambda) b) \mid \\
\leq & \frac{(b-a)^{3}}{120}\left[(1-\lambda)^{4}\left|h^{\prime \prime \prime}(a)\right|+4\left(\lambda^{4}+(1-\lambda)^{4}\right)\left|h^{\prime \prime \prime}(\lambda a+(1-\lambda) b)\right|+\lambda^{4}\left|h^{\prime \prime \prime}(b)\right|\right] .
\end{aligned}
$$

Corollary 3. If $q=1$ in (6), then

$$
\begin{aligned}
\mid \frac{1}{b-a} \int_{a}^{b} h(t) \mathrm{d} t & -\frac{3 \lambda^{2}-3 \lambda+1}{6}(b-a)^{2} h^{\prime \prime}(\lambda a+(1-\lambda) b)-\frac{2 \lambda-1}{2}(b-a) h^{\prime}(\lambda a+(1-\lambda) b) \\
& -h(\lambda a+(1-\lambda) b) \mid \leq \frac{(b-a)^{3}}{36}\left[\left(2 \lambda^{3}-2(1-\lambda)^{3}-3(1-\lambda)^{2}\right.\right. \\
& \left.-6(1-\lambda)-6 \ln \lambda)\left|h^{\prime \prime \prime}(a)\right|+\left(2(1-\lambda)^{3}-2 \lambda^{3}-3 \lambda^{2}-6 \lambda-6 \ln (1-\lambda)\right)\left|h^{\prime \prime \prime}(b)\right|\right] .
\end{aligned}
$$


Theorem 5. Let $h: I \subseteq[0, \infty) \rightarrow \mathbb{R}$ be a three times differentiable function on $I, a, b \in I$ with $a<b$, $h^{\prime \prime \prime} \in L_{1}([a, b])$, and $\lambda \in[0,1]$. If $\left|h^{\prime \prime \prime}(t)\right|^{q}$ is an extended s-convex function in the second sense on $[a, b]$, $s \in(-1,1]$, and $q \geq 1$, then

$$
\begin{aligned}
\mid \frac{1}{b-a} \int_{a}^{b} h(t) \mathrm{d} t & -\frac{3 \lambda^{2}-3 \lambda+1}{6}(b-a)^{2} h^{\prime \prime}(\lambda a+(1-\lambda) b) \\
& -\frac{2 \lambda-1}{2}(b-a) h^{\prime}(\lambda a+(1-\lambda) b)-h(\lambda a+(1-\lambda) b) \mid \leq \frac{(b-a)^{3}}{6}\left(\frac{1}{3 q+s+1}\right)^{1 / q} \\
& \times\left\{(1-\lambda)^{4}\left[\left|h^{\prime \prime \prime}(\lambda a+(1-\lambda) b)\right|^{q}+(3 q+s+1) B(3 q+1, s+1)\left|h^{\prime \prime \prime}(a)\right|^{q}\right]^{1 / q}\right. \\
& \left.+\lambda^{4}\left[\left|h^{\prime \prime \prime}(\lambda a+(1-\lambda) b)\right|^{q}+(3 q+s+1) B(3 q+1, s+1)\left|h^{\prime \prime \prime}(b)\right|^{q}\right]^{1 / q}\right\}
\end{aligned}
$$

where

$$
B(m, n)=\int_{0}^{1} t^{m-1}(1-t)^{n-1} \mathrm{~d} t, \quad m>0, \quad n>0
$$

is the classical beta function.

Proof. Since $\left|h^{\prime \prime \prime}(t)\right|^{q}$ is an extended s-convex function in the second sense on $[a, b]$, then by Lemma 1 and the Hölder inequality, we have

$$
\begin{aligned}
\mid & \frac{1}{b-a} \int_{a}^{b} h(t) \mathrm{d} t-\frac{3 \lambda^{2}-3 \lambda+1}{6}(b-a)^{2} h^{\prime \prime}(\lambda a+(1-\lambda) b) \\
& -\frac{2 \lambda-1}{2}(b-a) h^{\prime}(\lambda a+(1-\lambda) b)-h(\lambda a+(1-\lambda) b) \mid \\
\leq & \frac{(b-a)^{3}}{6}\left[\lambda^{4} \int_{0}^{1}\left|t^{3} h^{\prime \prime \prime}(t(\lambda a+(1-\lambda) b)+(1-t) b)\right| \mathrm{d} t\right. \\
& \left.+(1-\lambda)^{4} \int_{0}^{1}\left|t^{3} h^{\prime \prime \prime}(t(\lambda a+(1-\lambda) b)+(1-t) a)\right| \mathrm{d} t\right] \\
\leq & \frac{(b-a)^{3}}{6}\left\{\lambda^{4}\left(\int_{0}^{1} 1 \mathrm{~d} t\right)^{1-1 / q}\left[\int_{0}^{1} t^{3 q}\left|h^{\prime \prime \prime}(t(\lambda a+(1-\lambda) b)+(1-t) b)\right|^{q} \mathrm{~d} t\right]^{1 / q}\right. \\
& \left.+(1-\lambda)^{4}\left(\int_{0}^{1} 1 \mathrm{~d} t\right){ }^{1-1 / q}\left[\int_{0}^{1} t^{3 q}\left|h^{\prime \prime \prime}(t(\lambda a+(1-\lambda) b)+(1-t) a)\right|^{q} \mathrm{~d} t\right]^{1 / q}\right\} \\
\leq & \frac{(b-a)^{3}}{6}\left\{\lambda^{4}\left[\int_{0}^{1} t^{3 q}\left(t^{s}\left|h^{\prime \prime \prime}(\lambda a+(1-\lambda) b)\right|^{q}+(1-t)^{s}\left|h^{\prime \prime \prime}(b)\right|^{q}\right) \mathrm{d} t\right]^{1 / q}\right. \\
& \left.+(1-\lambda)^{4}\left[\int_{0}^{1} t^{3 q}\left(t^{s}\left|h^{\prime \prime \prime}(\lambda a+(1-\lambda) b)\right|^{q}+(1-t)^{s}\left|h^{\prime \prime \prime}(a)\right|^{q}\right) \mathrm{d} t\right]^{1 / q}\right\} \\
\leq & \frac{(b-a)^{3}}{6}\left(\frac{1}{3 q+s+1}\right)^{1 / q}\left\{( 1 - \lambda ) ^ { 4 } \left[\left|h^{\prime \prime \prime}(\lambda a+(1-\lambda) b)\right|^{q}\right.\right. \\
& +(b-a)^{3} \\
& +\lambda\left[\lambda^{4}\left[\frac{1}{3 q+s+1}\left|h^{\prime \prime \prime}(\lambda a+(1-\lambda) b)\right|^{q}+B(3 q+1, s+1)\left|h^{\prime \prime \prime}(b)\right|^{q}\right]^{1 / q}\right. \\
& \left.\left.+\frac{1}{3 q+s+1}\left|h^{\prime \prime \prime}(\lambda a+(1-\lambda) b)\right|^{q}+B(3 q+1, s+1)\left|h^{\prime \prime \prime}(a)\right|^{q}\right]^{1 / q}\right\}
\end{aligned}
$$

The proof of Theorem 5 is complete. 
Corollary 4. Under assumptions of Theorem 5 , if $s=1$, then

$$
\begin{array}{rl}
\mid \frac{1}{b-a} \int_{a}^{b} & h(t) \mathrm{d} t-\frac{3 \lambda^{2}-3 \lambda+1}{6}(b-a)^{2} h^{\prime \prime}(\lambda a+(1-\lambda) b) \\
& -\frac{2 \lambda-1}{2}(b-a) h^{\prime}(\lambda a+(1-\lambda) b)-h(\lambda a+(1-\lambda) b) \mid \\
\leq & \frac{(b-a)^{3}}{6}\left[\frac{1}{(3 q+1)(3 q+2)}\right]^{1 / q}\left\{(1-\lambda)^{4}\left[(3 q+1)\left|h^{\prime \prime \prime}(\lambda a+(1-\lambda) b)\right|^{q}+\left|h^{\prime \prime \prime}(a)\right|^{q}\right]^{1 / q}\right. \\
& \left.+\lambda^{4}\left[(3 q+1)\left|h^{\prime \prime \prime}(\lambda a+(1-\lambda) b)\right|^{q}+\left|h^{\prime \prime \prime}(b)\right|^{q}\right]^{1 / q}\right\} .
\end{array}
$$

Theorem 6. Let $h: I \subseteq[0, \infty) \rightarrow \mathbb{R}$ be a three times differentiable function on $I, a, b \in I$ with $a<b$, $h^{\prime \prime \prime} \in L_{1}([a, b])$, and $\lambda \in[0,1]$. If $\left|h^{\prime \prime \prime}(t)\right|^{q}$ is an extended s-convex function in the second sense on $[a, b]$, $s \in(-1,1]$, and $q>1$, then

$$
\begin{aligned}
\mid \frac{1}{b-a} \int_{a}^{b} h(t) & \mathrm{d} t-\frac{3 \lambda^{2}-3 \lambda+1}{6}(b-a)^{2} h^{\prime \prime}(\lambda a+(1-\lambda) b) \\
& -\frac{2 \lambda-1}{2}(b-a) h^{\prime}(\lambda a+(1-\lambda) b)-h(\lambda a+(1-\lambda) b) \mid \\
\leq & \frac{(b-a)^{3}}{6}\left(\frac{q-1}{4 q-1}\right)^{1-1 / q}\left(\frac{1}{s+1}\right)^{1 / q}\left\{(1-\lambda)^{4}\left[\left|h^{\prime \prime \prime}(\lambda a+(1-\lambda) b)\right|^{q}+\left|h^{\prime \prime \prime}(a)\right|^{q}\right]^{1 / q}\right. \\
& \left.+\lambda^{4}\left[\left|h^{\prime \prime \prime}(\lambda a+(1-\lambda) b)\right|^{q}+\left|h^{\prime \prime \prime}(b)\right|^{q}\right]^{1 / q}\right\} .
\end{aligned}
$$

Proof. Since $\left|h^{\prime \prime \prime}(t)\right|^{q}$ is an extended s-convex function in the second sense on $[a, b]$, then by Lemma 1 and the Hölder inequality, we have

$$
\begin{aligned}
\mid & \frac{1}{b-a} \int_{a}^{b} h(t) \mathrm{d} t-\frac{3 \lambda^{2}-3 \lambda+1}{6}(b-a)^{2} h^{\prime \prime}(\lambda a+(1-\lambda) b) \\
& -\frac{2 \lambda-1}{2}(b-a) h^{\prime}(\lambda a+(1-\lambda) b)-h(\lambda a+(1-\lambda) b) \mid \\
\leq & \frac{(b-a)^{3}}{6}\left[\lambda^{4} \int_{0}^{1}\left|t^{3} h^{\prime \prime \prime}(t(\lambda a+(1-\lambda) b)+(1-t) b)\right| \mathrm{d} t\right. \\
& \left.+(1-\lambda)^{4} \int_{0}^{1}\left|t^{3} h^{\prime \prime \prime}(t(\lambda a+(1-\lambda) b)+(1-t) a)\right| \mathrm{d} t\right] \\
\leq & \frac{(b-a)^{3}}{6}\left\{\lambda^{4}\left(\int_{0}^{1} t^{3 q /(q-1)} \mathrm{d} t\right)^{1-1 / q}\left[\int_{0}^{1}\left|h^{\prime \prime \prime}(t(\lambda a+(1-\lambda) b)+(1-t) b)\right|^{q} \mathrm{~d} t\right]^{1 / q}\right. \\
& \left.+(1-\lambda)^{4}\left(\int_{0}^{1} t^{3 q /(q-1)} \mathrm{d} t\right)^{1-1 / q}\left[\int_{0}^{1}\left|h^{\prime \prime \prime}(t(\lambda a+(1-\lambda) b)+(1-t) a)\right|^{q} \mathrm{~d} t\right]^{1 / q}\right\} \\
\leq & \frac{(b-a)^{3}}{6}\left\{\lambda^{4}\left(\int_{0}^{1} t^{3 q /(q-1)} \mathrm{d} t\right)^{1-1 / q}\left[\int_{0}^{1}\left(t^{s}\left|h^{\prime \prime \prime}(\lambda a+(1-\lambda) b)\right|^{q}+(1-t)^{s}\left|h^{\prime \prime \prime}(b)\right|^{q}\right) \mathrm{d} t\right]^{1 / q}\right. \\
& \left.+(1-\lambda)^{4}\left(\int_{0}^{1} t^{3 q /(q-1)} \mathrm{d} t\right)^{1-1 / q}\left[\int_{0}^{1}\left(t^{s}\left|h^{\prime \prime \prime}(\lambda a+(1-\lambda) b)\right|^{q}+(1-t)^{s}\left|h^{\prime \prime \prime}(a)\right|^{q}\right) \mathrm{d} t\right]^{1 / q}\right\} \\
= & \frac{(b-a)^{3}}{6}\left(\frac{q-1}{4 q-1}\right)^{1-1 / q}\left(\frac{1}{s+1}\right)^{1 / q}\left\{(1-\lambda)^{4}\left[\left|h^{\prime \prime \prime}(\lambda a+(1-\lambda) b)\right|^{q}+\left|h^{\prime \prime \prime}(a)\right|^{q}\right]^{1 / q}\right. \\
& \left.+\lambda^{4}\left[\left|h^{\prime \prime \prime}(\lambda a+(1-\lambda) b)\right|^{q}+\left|h^{\prime \prime \prime}(b)\right|^{q}\right]^{1 / q}\right\} .
\end{aligned}
$$

The proof of Theorem 6 is complete. 
Corollary 5. Under assumptions of Theorem 6 , if $s=1$, then

$$
\begin{aligned}
\mid \frac{1}{b-a} \int_{a}^{b} h(t) \mathrm{d} t- & \frac{3 \lambda^{2}-3 \lambda+1}{6}(b-a)^{2} h^{\prime \prime}(\lambda a+(1-\lambda) b) \\
& -\frac{2 \lambda-1}{2}(b-a) h^{\prime}(\lambda a+(1-\lambda) b)-h(\lambda a+(1-\lambda) b) \mid \\
= & \frac{(b-a)^{3}}{6}\left(\frac{q-1}{4 q-1}\right)^{1-1 / q}\left(\frac{1}{2}\right)^{1 / q}\left\{(1-\lambda)^{4}\left[\left|h^{\prime \prime \prime}(\lambda a+(1-\lambda) b)\right|^{q}+\left|h^{\prime \prime \prime}(a)\right|^{q}\right]^{1 / q}\right. \\
& \left.+\lambda^{4}\left[\left|h^{\prime \prime \prime}(\lambda a+(1-\lambda) b)\right|^{q}+\left|h^{\prime \prime \prime}(b)\right|^{q}\right]^{1 / q}\right\} .
\end{aligned}
$$

\section{Applications to Means}

Making use of results for integral inequalities of the Hermite-Hadmard type for extended s-convex functions in the second sense in the above section, we construct some inequalities for means.

Let $b>a>0$, the arithmetic mean $A_{\mu}(a, b)$ and the generalized logarithmic mean $L_{r}(a, b)$ are defined $[28,29]$ respectively as

$$
A_{\mu}(a, b)=\mu a+(1-\mu) b, \quad \mu \in[0,1]
$$

and

$$
L_{r}(a, b)= \begin{cases}{\left[\frac{b^{r+1}-a^{r+1}}{(r+1)(b-a)}\right]^{1 / r},} & r \neq 0,-1 ; \\ \frac{b-a}{\ln b-\ln a^{\prime}} & r=-1 ; \\ \frac{1}{e}\left(\frac{b^{b}}{a^{a}}\right)^{1 /(b-a)}, & p=0 .\end{cases}
$$

Let $h(t)=\frac{t^{s+3}}{(s+1)(s+2)(s+3)}$. Then $h^{\prime \prime \prime}(t)=t^{s}$. When $u, v>0, \mu \in(0,1)$, if $q \geq 1,-1<s q \leq 1$, and $-1<s \leq 1$, then

$$
\left|h^{\prime \prime \prime}(\mu u+(1-\mu) v)\right|^{q}=\left|[\mu u+(1-\mu) v]^{s}\right|^{q} \leq \mu^{s}\left|h^{\prime \prime \prime}(u)\right|^{q}+(1-\mu)^{s}\left|h^{\prime \prime \prime}(v)\right|^{q}
$$

which means that $\left|h^{\prime \prime \prime}(t)\right|^{q}=t^{s q}$ is an extended $s$-convex function in the second sense on $[a, b]$. Meanwhile,

$$
\frac{1}{b-a} \int_{a}^{b} h(t) \mathrm{d} t=\frac{1}{(s+1)(s+2)(s+3)} L_{s+3}^{s+3}(a, b) .
$$

Consequently, applying (5) in Theorem 4 to $\left|h^{\prime \prime \prime}(t)\right|^{q}=t^{s q}$ yields the following theorem.

Theorem 7. Let $b>a>0, q \geq 1,-1<s q \leq 1,-1<s \leq 1$, and $\lambda \in[0,1]$. Then

$$
\begin{aligned}
\mid L_{s+3}^{s+3}(a, b) & -\frac{(s+2)(s+3)\left(3 \lambda^{2}-3 \lambda+1\right)}{6}(b-a)^{2} A_{\lambda}^{s+1}(a, b) \\
& -\frac{(s+3)(2 \lambda-1)}{2}(b-a) A_{\lambda}^{s+2}(a, b)-A_{\lambda}^{s+3}(a, b) \mid \leq \frac{(b-a)^{3}}{24}\left(\frac{4}{s+4}\right)^{1 / q} \\
& \times[(s+1)(s+2)(s+3)]^{1-1 / q}\left\{(1-\lambda)^{4}\left[(s+1)(s+2)(s+3)\left|A_{\lambda}(a, b)\right|^{s q}+6 a^{s q}\right]^{1 / q}\right. \\
& \left.+\lambda^{4}\left[(s+1)(s+2)(s+3)\left|A_{\lambda}(a, b)\right|^{s q}+6 b^{s q}\right]^{1 / q}\right\} .
\end{aligned}
$$

Corollary 6. Under assumptions of Theorem 7 , if $\lambda=\frac{1}{2}$ and $s=1$, then

$$
\begin{aligned}
\mid L_{s+3}^{s+3}(a, b)- & A_{1 / 2}^{s+3}(a, b)-\frac{(b-a)^{2}}{2} A_{1 / 2}^{s+1}(a, b) \mid \\
\leq & \frac{(b-a)^{3}}{16}\left(\frac{1}{5}\right)^{1 / q}\left\{\left[4 A_{1 / 2}^{s q}(a, b)+a^{s q}\right]^{1 / q}+\left[4 A_{1 / 2}^{s q}(a, b)+b^{s q}\right]^{1 / q}\right\}
\end{aligned}
$$


Applying Theorem 5 to the function $\left|h^{\prime \prime \prime}(t)\right|^{q}=t^{s q}$ yields the following theorem.

Theorem 8. Let $b>a>0, q \geq 1,-1<s q \leq 1,-1<s \leq 1$, and $\lambda \in[0,1]$. Then

$$
\begin{gathered}
\mid L_{s+3}^{s+3}(a, b)-\frac{(s+2)(s+3)\left(3 \lambda^{2}-3 \lambda+1\right)}{6}(b-a)^{2} A_{\lambda}^{s+1}(a, b) \\
-\frac{(s+3)(2 \lambda-1)}{2}(b-a) A_{\lambda}^{s+2}(a, b)-A_{\lambda}^{s+3}(a, b) \mid \\
\leq \frac{(b-a)^{3}(s+1)(s+2)(s+3)}{6}\left(\frac{1}{3 q+s+1}\right)^{1 / q} \\
\times\left\{(1-\lambda)^{4}\left[\left|A_{\lambda}(a, b)\right|^{s q}+(3 q+s+1) B(3 q+1, s+1) a^{s q}\right]^{1 / q}\right. \\
\left.\quad+\lambda^{4}\left[\left|A_{\lambda}(a, b)\right|^{s q}+(3 q+s+1) B(3 q+1, s+1) b^{s q}\right]^{1 / q}\right\} .
\end{gathered}
$$

Corollary 7. Under assumptions of Theorem 8 , if $\lambda=\frac{1}{2}$ and $s=1$, then

$$
\begin{aligned}
\left|L_{s+3}^{s+3}(a, b)-A_{1 / 2}^{s+3}(a, b)-\frac{(b-a)^{2}}{2} A_{1 / 2}^{s+1}(a, b)\right| \leq \frac{(b-a)^{3}}{4}\left[\frac{1}{(3 q+1)(3 q+2)}\right]^{1 / q} & \\
& \times\left\{\left[(3 q+1) A_{1 / 2}^{s q}(a, b)+a^{s q}\right]^{1 / q}+\left[(3 q+1) A_{1 / 2}^{s q}(a, b)+b^{s q}\right]^{1 / q}\right\} .
\end{aligned}
$$

Applying Theorem 6 to $\left|h^{\prime \prime \prime}(t)\right|^{q}=t^{s q}$ yields

Theorem 9. Let $b>a>0, q>1,-1<s q \leq 1,-1<s \leq 1$, and $\lambda \in[0,1]$. Then

$$
\begin{aligned}
& \mid L_{s+3}^{s+3}(a, b)-\frac{(s+2)(s+3)\left(3 \lambda^{2}-3 \lambda+1\right)}{6}(b-a)^{2} A_{\lambda}^{s+1}(a, b) \\
& -\frac{(s+3)(2 \lambda-1)}{2}(b-a) A_{\lambda}^{s+2}(a, b)-A_{\lambda}^{s+3}(a, b) \mid \\
& \leq \frac{(b-a)^{3}(s+1)(s+2)(s+3)}{6}\left(\frac{q-1}{4 q-1}\right)^{1-1 / q}\left(\frac{1}{s+1}\right)^{1 / q} \\
& \quad \times\left\{(1-\lambda)^{4}\left[\left|A_{\lambda}(a, b)\right|^{s q}+a^{s q}\right]^{1 / q}+\lambda^{4}\left[\left|A_{\lambda}(a, b)\right|^{s q}+b^{s q}\right]^{1 / q}\right\} .
\end{aligned}
$$

Corollary 8. Under assumptions of Theorem 9 , if $\lambda=\frac{1}{2}$ and $s=1$, then

$$
\begin{aligned}
\mid L_{s+3}^{s+3}(a, b)- & A_{1 / 2}^{s+3}(a, b)-\frac{(b-a)^{2}}{2} A_{1 / 2}^{s+1}(a, b) \mid \\
\leq & \frac{(b-a)^{3}}{4}\left(\frac{q-1}{4 q-1}\right)^{1-1 / q}\left(\frac{1}{2}\right)^{1 / q}\left\{\left[A_{1 / 2}^{s q}(a, b)+a^{s q}\right]^{1 / q}+\left[A_{1 / 2}^{s q}(a, b)+b^{s q}\right]^{1 / q}\right\} .
\end{aligned}
$$

Remark 3. Since the identity (3) in [6] and the newly-established identity (4) in Lemma 1 can not be derived from each other, we can be sure that all results in this paper and those in [6] can not be compared with each other.

Author Contributions: Both authors contributed equally in writing this article. Both authors read and approved the final manuscript.

Funding: This research was funded by the Fostering Project for Successfully Applying for the National Natural Science Foundation of China at the Inner Mongolia University for Nationalities (Grant No. NMDGP17104), by the Natural Science Foundation of Inner Mongolia Autonomous Region of China (Grant No. 2018LH01002), and by the Science Research Fund of Inner Mongolia University for Nationalities in China (Grant No. NMDYB17157).

Acknowledgments: The authors are thankful to Bo-Yan Xi for his academic and technical help in preparing and revising the original version of this paper.

Conflicts of Interest: The authors declare no conflict of interest. 


\section{References}

1. Niculescu, C.P.; Persson, L.-E. Convex Functions and Their Applications: A Contemporary Approach. In CMS Books in Mathematics/Ouvrages de Mathématiques de la SMC, 2nd ed.; Springer: Cham, Switzerland, 2018.

2. Pečarić, J.; Proschan, F.; Tong, Y.L. Convex Functions, Partial Orderings, and Statistical Applications; Academic Press: Cambridge, MA, USA, 1992.

3. Dragomir, S.S.; Pearce, C.E.M. Selected Topics on Hermite-Hadamard Type Inequalities and Applications; Amended Version, RGMIA Monographs; Victoria University: Victoria, Australia, 2002.

4. Hudzik, H.; Maligranda, L. Some remarks on s-convex functions. Aequal. Math. 1994, 48, 100-111. [CrossRef]

5. Xi, B.-Y.; Qi, F. Inequalities of Hermite-Hadamard type for extended s-convex functions and applications to means. J. Nonlinear Convex Anal. 2015, 16, 873-890.

6. Chun, L.; Qi, F. Inequalities of Simpson type for functions whose third derivatives are extended s-convex functions and applications to means. J. Comput. Anal. Appl. 2015, 19, 555-569.

7. Hua, J.; Xi, B.-Y.; Qi, F. Hermite-Hadamard type inequalities for geometric-arithmetically s-convex functions. Commun. Korean Math. Soc. 2014, 29, 51-63. [CrossRef]

8. Hua, J.; Xi, B.-Y.; Qi, F. Inequalities of Hermite-Hadamard type involving an s-convex function with applications. Appl. Math. Comput. 2014, 246, 752-760. [CrossRef]

9. Hua, J.; Xi, B.-Y.; Qi, F. Some new inequalities of Simpson type for strongly s-convex functions. Afr. Mater. 2015, 26, 741-752. [CrossRef]

10. Chun, L.; Qi, F. Integral inequalities of Hermite-Hadamard type for functions whose 3rd derivatives are s-convex. Appl. Math. 2012, 3, 1680-1685. [CrossRef]

11. Shuang, Y.; Yin, H.-P.; Qi, F. Hermite-Hadamard type integral inequalities for geometric-arithmetically s-convex functions. Analysis 2013, 33, 197-208. [CrossRef]

12. Sun, J.; Xi, B.-Y.; Qi, F. Some new inequalities of the Hermite-Hadamard type for extended s-convex functions. J. Comput. Anal. Appl. 2019, 26, 985-996.

13. Wang, S.-H.; Qi, F. Hermite-Hadamard type inequalities for s-convex functions via Riemann-Liouville fractional integrals. J. Comput. Anal. Appl. 2017, 22, 1124-1134.

14. Xi, B.-Y.; Gao, D.-D.; Qi, F. Integral Inequalities of Hermite-Hadamard Type for $(\alpha, s)$-Convex and $(\alpha, s, m)$-Convex Functions. HAL Archives. 2018. Available online: https://hal.archives-ouvertes.fr/hal01761678 (accessed on 24 October 2018).

15. Xi, B.-Y.; Hua, J.; Qi, F. Hermite-Hadamard type inequalities for extended s-convex functions on the co-ordinates in a rectangle. J. Appl. Anal. 2014, 20, 29-39. [CrossRef]

16. Kirmaci, U.S.; Bakula, M.K.; Özdemir, M.E.; Pečarić, J. Hadamard-type inequalities for s-convex functions. Appl. Math. Comput. 2007, 193, 26-35. [CrossRef]

17. Hussain, S.; Bhatti, M.I.; Iqbal, M. Hadamard-type inequalities for s-convex functions I. Punjab Univ. J. Math. 2009, 41, 51-60.

18. Sarikaya, M.Z.; Set, E.; Özdemir, M.E. On new inequalities of Simpson's type for s-convex functions. Comput. Math. Appl. 2010, 60, 2191-2199. [CrossRef]

19. Abbaszadeh, S.; Ebadian, A. Nonlinear integrals and Hadamard-type inequalities. Soft Comput. 2018, 22, 2843-2849. [CrossRef]

20. Dragomir, S.S.; Fitzpatrick, S. The Hadamard's inequality for s-convex functions in the second sense. Demonstration Math. 1999, 32, 687-696.

21. Alomari, M.; Darus, M.; Dragomir, S.S.; Cerone, P. Ostrowski type inequalities for functions whose derivatives are s-convex in the second sense. Appl. Math. Lett. 2010, 23, 1071-1076. [CrossRef]

22. Alomari, M.W.; Darus, M.; Kirmaci, U.S. Some inequalities of Hermite-Hadamard type for s-convex functions. Acta Math. Sci. Ser. B Engl. Ed. 2011, 31, 1643-1652. [CrossRef]

23. Avci, M.; Kavurmaci, H.; Özdemir, M.E. New inequalities of Hermite-Hadamard type via s-convex functions in the second sense with applications. Appl. Math. Comput. 2011, 217, 5171-5176. [CrossRef]

24. Bai, S.-P.; Wang, S.-H.; Qi, F. Some Hermite-Hadamard type inequalities for $n$-time differentiable $(\alpha, m)$-convex functions. J. Inequal. Appl. 2012, 2012. [CrossRef]

25. Chun, L.; Qi, F. Integral inequalities of Hermite-Hadamard type for functions whose third derivatives are convex. J. Inequal. Appl. 2013, 2013. [CrossRef] 
26. Qi, F.; Wei, Z.-L.; Yang, Q. Generalizations and refinements of Hermite-Hadamard's inequality. Rocky Mt. J. Math. 2005, 35, 235-251. [CrossRef]

27. Wang, S.-H.; Xi, B.-Y.; Qi, F. Some new inequalities of Hermite-Hadamard type for $n$-time differentiable functions which are $m$-convex. Analysis 2012, 32, 247-262. [CrossRef]

28. Bullen, P.S. Handbook of Means and Their Inequalities. In Mathematics and Its Applications; Kluwer Academic Publishers: Dordrecht, The Netherlands; Boston, MA, USA; London, UK, 2003; Volume 560.

29. Qi, F.; Lim, D. Integral representations of bivariate complex geometric mean and their applications. J. Comput. Appl. Math. 2018, 330, 41-58. [CrossRef]

(C) 2018 by the authors. Licensee MDPI, Basel, Switzerland. This article is an open access article distributed under the terms and conditions of the Creative Commons Attribution (CC BY) license (http:/ / creativecommons.org/licenses/by/4.0/). 